\title{
Diurnal Variation of the Planetary Boundary Layer Height Observed from GNSS Radio Occultation and Radiosonde Soundings over the Southern Great Plains
}

\author{
Kevin J. Nelson, ${ }^{\mathrm{a}}$ Feigin Xie, ${ }^{\mathrm{a}}$ Chi O. Ao, ${ }^{\mathrm{b}}$ And Mayra I. Oyola-Merced ${ }^{\mathrm{b}}$ \\ ${ }^{a}$ Texas A\&M University-Corpus Christi, Corpus Christi, Texas \\ ${ }^{\mathrm{b}}$ Jet Propulsion Laboratory, California Institute of Technology, Pasadena, California
}

(Manuscript received 28 November 2020, in final form 1 September 2021)

\begin{abstract}
The planetary boundary layer (PBL) height (PBLH) is a key physical parameter of the PBL affected by numerous physical processes within the boundary layer. Specifically, the PBLH over land exhibits large spatial and temporal variation across different geographical regions. In this study, the Constellation Observing System for Meteorology, Ionosphere and Climate (COSMIC) radio occultation (RO) profiles and high-resolution radiosonde profiles from 2007 to 2013 were analyzed to estimate the diurnal cycle of the PBLH over the Southern Great Plains (SGP) in the United States. Large variations in PBLH derived from radiosonde temperature, moisture, and refractivity are observed on seasonal scales. COSMIC RO is capable of observing diurnal and seasonal variations in the terrestrial PBLH over the SGP region. Annual mean diurnal amplitude of approximately $250 \mathrm{~m}$ in the terrestrial PBLH was observed, with maxima occurring at around 1500 local solar time (LST) in both the collocated radiosondes and COSMIC RO profiles. Seasonal changes in the PBLH diurnal cycles ranging from approximately 100 to $400 \mathrm{~m}$ were also observed. Such PBL diurnal and seasonal changes can be further incorporated into PBL parameterizations to help improve weather and climate model prediction.
\end{abstract}

SIGNIFICANCE STATEMENT: The atmospheric planetary boundary layer (PBL) and its height (PBLH) control many atmospheric processes that affect our everyday lives. Observations of the PBL are usually limited to radiosondes at limited time intervals. GNSS radio occultation (RO) provide high-vertical-resolution atmospheric observations that are ideal for PBL study. This study demonstrates that the GNSS RO is capable of capturing the diurnal and seasonal variations of the PBLH over the southern Great Plains (SGP) well as compared to the collocated radiosonde observations

KEYWORDS: Boundary layer; Global positioning systems (GPS); Remote sensing; Diurnal effects

\section{Introduction}

The planetary boundary layer (PBL) constitutes the lowest layer of the atmosphere closest to Earth's surface where turbulent processes typically dominate the vertical distribution of heat, momentum, and particulate matter (e.g., Lee 2018; Stull 1988). The PBL height (PBLH) is a key parameter for formulations of many multiscale processes. Consequently, the PBL can also impact global energy and water cycles on climatological scales (e.g., Klein and Hartmann 1993; Ramanathan et al. 1989). It is expected that changes in the PBL on the diurnal scale are more prominent over land, and less so over water or ice where seasonal variation tends to dominate PBL variation (Seidel et al. 2012; Sawyer and Li 2013). However, a lack of understanding and observations of PBL processes leads to significant uncertainties in weather and climate model forecasts (e.g., Jordan et al. 2010; McGibbon and Bretherton 2019; Nuijens and Siebesma 2019; Palm et al. 2005).

The characteristics of the PBL change often, and sometimes rapidly, on various spatial and temporal scales. As a result, boundary layer physics parameterizations in models can result in poor treatment of convection, clouds, and precipitation (Holtslag et al. 2013). However, some of the most distinct

Corresponding author: Kevin J. Nelson, knelson12@islander. tamucc.edu changes occur on diurnal time scales, and as such, the variability of the PBL tends to be dominated by diurnal processes (e.g., Garratt 1994; Stull 1988). Without the influence of synoptic-scale systems, the PBL is usually less than $500 \mathrm{~m}$ deep during nighttime due to surface radiational cooling. During the daytime, the PBL can be significantly deeper due to surface heating and the resulting convection. Liu and Liang (2010) created a global climatology of PBLH derived from field campaign observations and showed that the PBLH over both terrestrial and maritime regions have a strong diurnal cycle, with PBLH reaching its highest value at 1500 local solar time (LST) for terrestrial boundary layers and at 1200 LST for marine boundary layers. The PBL and corresponding PBLH have also been shown to be affected by both synopticand short-term climate-scale events such that the amplitude of the diurnal cycle of the PBLH is amplified. While one segment of the diurnal cycle is amplified, the opposite segment PBL and PBLH can remain mostly unchanged or become damped (Zhang et al. 2020). The local PBL has also been shown to be modified by microfronts, particularly in the overnight hours, which can significantly modulate boundary layer processes and affect the PBLH (Mahrt 1999).

In the past, comprehensive study of the global PBL has been hindered by a lack of spatially diverse observations. Most PBL observations over land are restricted to a few extensive field campaigns [e.g., Innovative Strategies for 
Observations in the Arctic Atmospheric Boundary Layer (ISOBAR); Kral et al. 2018; Experimental Planetary Boundary Layer Instrumentation Assessment (XPIA); Lundquist et al. 2017; Boundary Layer Experiment 1996 (BLX96); Stull et al. 1997], which are extremely useful, but are limited in both space and time. Sparse observations over open oceans also pose a significant problem and are again limited to marine boundary layer (MBL) field campaigns [e.g., ASTEX; Albrecht et al. 1995; VAMOS Ocean-Cloud-AtmosphereLand Study Regional Experiment (VOCALS-REx); Wood et al. 2011]. Diurnal observation studies of the PBL over both land and ocean, while helpful, are similarly limited in space to single stations (Basha and Ratnam 2009; Mehta et al. 2017). While the thickness of PBL can be as large as a few kilometers (Ao et al. 2012; von Engeln and Teixeira 2013; Zhou 2021), the transition layer from the PBL to the free troposphere across the PBLH is only a few tens to hundreds of meters, making it incredibly difficult to detect with conventional, lowvertical-resolution satellite passive microwave or infrared sounders (Maddy and Barnet 2008) or to simulate in both weather and climate models (e.g., Suarez et al. 1983; Wood and Bretherton 2004).

On the other hand, the Global Navigation Satellite System (GNSS) radio occultation (RO) profiling technique provides high-vertical-resolution ( $100 \mathrm{~m}$ in the lower troposphere) soundings necessary for PBLH detection over both land and ocean in all-weather conditions (Kursinski et al. 1997). GNSS $\mathrm{RO}$ data from the joint RO mission between Taiwan and the United States, the Formosa 3/Constellation Observing System for Meteorology, Ionosphere and Climate (hereafter COSMIC) launched in 2006, provided approximately 2000 occultations globally with quasi-random sampling in both space and time (Anthes et al. 2008). Furthermore, the six-satellite COSMIC constellation offers composite diurnal sampling capability. Additionally, implementation of open-loop signal tracking on COSMIC RO receivers and radio holographic retrieval allow for more accurate measurements of the PBL (Ao et al. 2009; Ganeshan and Wu 2015). With the advances in GNSS RO technology over the past few decades, PBL studies using GNSS RO have advanced significantly and have demonstrated good results (Ao et al. 2008, 2012; Sokolovskiy et al. 2006; Ware et al. 1996; Xie et al. 2010, 2012).

Using GNSS RO refractivity profiles and water vapor pressure profiles, Ao et al. (2012) developed a global climatology of marine PBLH. Other studies, such as Guo et al. (2011) and Sokolovskiy et al. (2011) have also shown that RO bending angle and refractivity are suitable for detecting the PBLH globally. Xie et al. (2012) demonstrated highly consistent PBLH observations from COSMIC with the high-resolution radiosondes from the VOCALS-REx field campaign (hereafter VOCALS; Wood et al. 2011) off the coast of the southeast Pacific, but with a small bias. Additional studies since then have shown good agreement between GNSS RO PBLH and radiosonde PBLH (Ganeshan and Wu 2015; Ho et al. 2015; Winning et al. 2017). Numerous studies have used GNSS RO to study the PBL over marine environments with low convective instability and distinct boundary layers (Ho et al. 2015; Winning et al. 2017; Xie et al. 2012). However, few RO studies have been carried out over land where the PBL exhibits much more complex spatial and temporal variations. This study examines the terrestrial PBL using COSMIC RO measurements. Knowing that GNSS RO sounding encounters difficulty penetrating deep into the lower troposphere near the surface (Ao et al. 2008; Sokolovskiy et al. 2006) and that high terrain can interfere with RO signal tracking, we choose the Southern Great Plains (SGP) as the study region with relatively flat terrain, and a high number of in situ observations that could be used to verify the GNSS RO measurements.

The remainder of this paper is structured as follows. Section 2 describes the datasets and methodologies used for PBL analysis. The unique properties of our study area and the limitations of our methodologies will also be discussed. Section 3 details PBL analysis based on 7 years of radiosonde measurements. In section 4, PBL analysis based on COSMIC GNSS RO measurements are carried out and compared with radiosonde observations. Section 5 summarizes the key findings and conclusions.

\section{Data and methodology}

\section{a. COSMIC RO profile data}

In this study, we used 7 years (2007-13) of COSMIC RO level-2 refractivity profiles (7683 in total) retrieved by the University Corporation for Atmospheric Research (UCAR) COSMIC Data Analysis and Archive Center (CDAAC). The level 2 postprocessed refractivity product (version 2010.2640) was used for this study, as the most recent reprocessed and postprocessed products (versions 2013.3520 and 2014.2860, respectively) have significantly fewer profiles reaching into the PBL due to changes in the quality control procedures used. Specifically, a signal-to-noise ratio (SNR) cutoff was introduced, resulting in fewer profiles penetrating into the PBL (e.g., the lowest $1-2 \mathrm{~km}$ ). This is a known issue and will be addressed with the next reprocessing of COSMIC and the follow-on COSMIC-2 mission profiles. COSMIC RO profiles and available radiosondes need to be collocated to each other in both space and time in order to be reasonably compared. A spatial threshold of $300 \mathrm{~km}$ and a temporal threshold of $\pm 3 \mathrm{~h}$ from the time of the radiosonde launch were chosen as the collocation criteria for this study based on results from tests done to optimize available samples while minimizing potential bias. It is worth noting that these criteria were selected specific to the needs of the study and the study region. Other projects with different needs or those in different locations with varying topography or other surface properties may necessitate or benefit from different collocation criteria.

Only profiles that meet the collocation criteria while simultaneously penetrating to within $500 \mathrm{~m}$ of the surface were ultimately considered. This amounts to a total of 1335 pairs of RO profiles and radiosondes. Note that the total number of COSMIC RO soundings started decreasing after 2010 as RO satellites reached the end of their lifespan. The level 2 refractivity profiles are given as a function of the geometric height 
above mean sea level (MSL). Due to the varying terrain heights in the SGP region, each RO refractivity profile was adjusted to MSL by subtracting the surface geometric height from the collocated radiosonde from each RO profile height. As such, all PBLH values derived from the RO profiles are referenced to the local terrain surface.

\section{b. Radiosonde profile data}

The Central Station $\left(\mathrm{C} 1 ; 36.60732^{\circ} \mathrm{N}, 97.48764^{\circ} \mathrm{W}\right)$ of the Atmospheric Radiation Measurement (ARM) Southern Great Plains launches standard Vaisala radiosondes every $6 \mathrm{~h}$ at 0530, 1130, 1820, and 2330 UTC. These radiosonde launches are treated as the next hour's radiosondes $(0000$, 0600, 1200, and 1800 UTC) because it takes approximately 30 min, which provide diurnal sampling of the thermodynamic state of the atmosphere. Seven years (2007-13) of SGP radiosondes were analyzed, totaling 9802 in all. Radiosondes from ancillary stations used during various field campaigns run at the SGP site over the course of the observation period are also included, which only have twice daily (0000 and 1200 UTC) soundings. These ancillary, twice-daily radiosondes account for approximately $1.6 \%$ of the total number of radiosondes.

Atmospheric refractivity in the lower troposphere is a function of pressure, temperature, and moisture. The refractivity profile for each radiosonde can be calculated using Eq. (1):

$$
N=b_{1} \frac{P}{T}+b_{2} \frac{P_{w}}{T^{2}}
$$

where $b_{1}=77.6 \mathrm{~K} \mathrm{hPa}^{-1}, P$ is atmospheric pressure $(\mathrm{hPa})$, $T$ is atmospheric temperature $(\mathrm{K})$, and $b_{2}=3.73 \times$ $10^{5} \mathrm{~K}^{2} \mathrm{hPa}^{-1}$ (Kursinski et al. 1997). To be consistent with the geometric heights in the COSMIC RO dataset, the geopotential heights used in radiosonde sounding are converted to geometric height above the MSL. Each radiosonde profile is interpolated to $10-\mathrm{m}$ vertical intervals using a cubic function and smoothed using three-point (30-m) smoothing to remove very fine structures. The PBLH values from radiosondes are also referenced to local terrain surface, similar to the RO-derived PBLH values. We acknowledge that long-term analyses of radiosonde data can potentially suffer from both spatial and temporal inhomogeneity-derived bias as described by Dai et al. (2002) and Zhou et al. (2021). Based on additional investigation of the data used for this study and its supporting documentation (Holdridge 2020) we believe that such inhomogeneities do not affect the PBLH detection for the purpose of this study based on the length of the radiosonde dataset used.

\section{c. Study region}

Figure 1a shows the study region centered around $\mathrm{C} 1$ of the ARM Southern Great Plains site (Sisterson et al. 2016) southeast of Lamont, Oklahoma, indicated by the star in the center of the map. Ancillary stations used during field campaigns for additional radiosondes are denoted by black, filled circles. Color-filled contours in Fig. 1a denote the terrain heights derived from the ECMWF model reanalysis version 5 (ERA5; Hersbach et al. 2020) surface geopotential at $0.25^{\circ} \times 0.25^{\circ}$ horizontal resolution. The terrain heights for majority of the SGP region are less than $600 \mathrm{~m}$ (approximately $65 \%$ of the domain), with higher values in western Oklahoma and Kansas. Solid contours in Fig. 1a show the fractional number of COSMIC RO profiles that reach $500 \mathrm{~m}$ above the local terrain height or below based on the 4607 COSMIC RO refractivity profiles from 2007 to 2013 as described in section $2 \mathrm{a}$. Within the study region, each $0.1^{\circ} \times 0.1^{\circ}$ grid has between 40 and $55 \mathrm{RO}$ profiles, with the highest concentration of profiles ( $\sim 65$ profiles) over the northwestern portion of the domain, and lowest concentration of profiles ( $\sim 30$ profiles) over the southeastern portion of the domain. Note that the fraction of deep penetrating RO profiles appears to be higher over the higher terrain area (e.g., the western portion of the domain). Figure $1 b$ further shows that approximately $85 \%$ of RO profiles reach below $500 \mathrm{~m}$ above the terrain, and 10\% reach Earth's surface.

\section{d. PBL height detection}

The PBLH, a key parameter separating the PBL from free troposphere above, can be identified using a wide array of methods, ranging from relatively simple methods such as identifying the height of a particular physical parameter in a vertical profile (Seidel et al. 2010; Xie et al. 2006, 2012) to analyzing the atmospheric electric field (Nicoll et al. 2018). Dynamical relationships for the PBLH can also be derived from incompressible Navier-Stokes equations and other complex dynamical relationships (Mahrt 1999, 2014; Zhang et al. 2011).

One of the simplest methods to use for identifying the PBLH is the gradient method. The method was initially introduced in the late 1980s by Stull (1988) and Oke (1988) using the level of the maximum vertical gradient of potential temperature indicating a transition between convectively less stable regions below a stable region above. A comprehensive survey of the various gradient methods used for determining PBLH can be found in Seidel et al. (2010).

Note that the GNSS RO refractivity is a function of temperature and moisture; thus, the refractivity gradient is a weighted sum of the temperature and moisture gradients (e.g., Ao et al. 2012). Generally, the PBLH corresponds to the height of the temperature inversion base, or near the height of the maximum (minimum) gradient of temperature (humidity or refractivity), respectively (Ao et al. 2012; Ganeshan and Wu 2015; Seidel et al. 2010; Winning et al. 2017; Xie et al. 2012). The same methodology can be applied to both the radiosonde and RO refractivity profiles for PBLH detection. Since different variables from the same sounding could lead to different PBLH, we introduce the subscripts to differentiate the PBLH identified from the maximum gradient of temperature $\left(\mathrm{PBLH}_{T}\right)$, minimum gradient of specific humidity $\left(\mathrm{PBLH}_{q}\right)$, and minimum gradient of refractivity $\left(\mathrm{PBLH}_{N}\right)$. It is worth noting that GNSS RO soundings encounter difficulty 

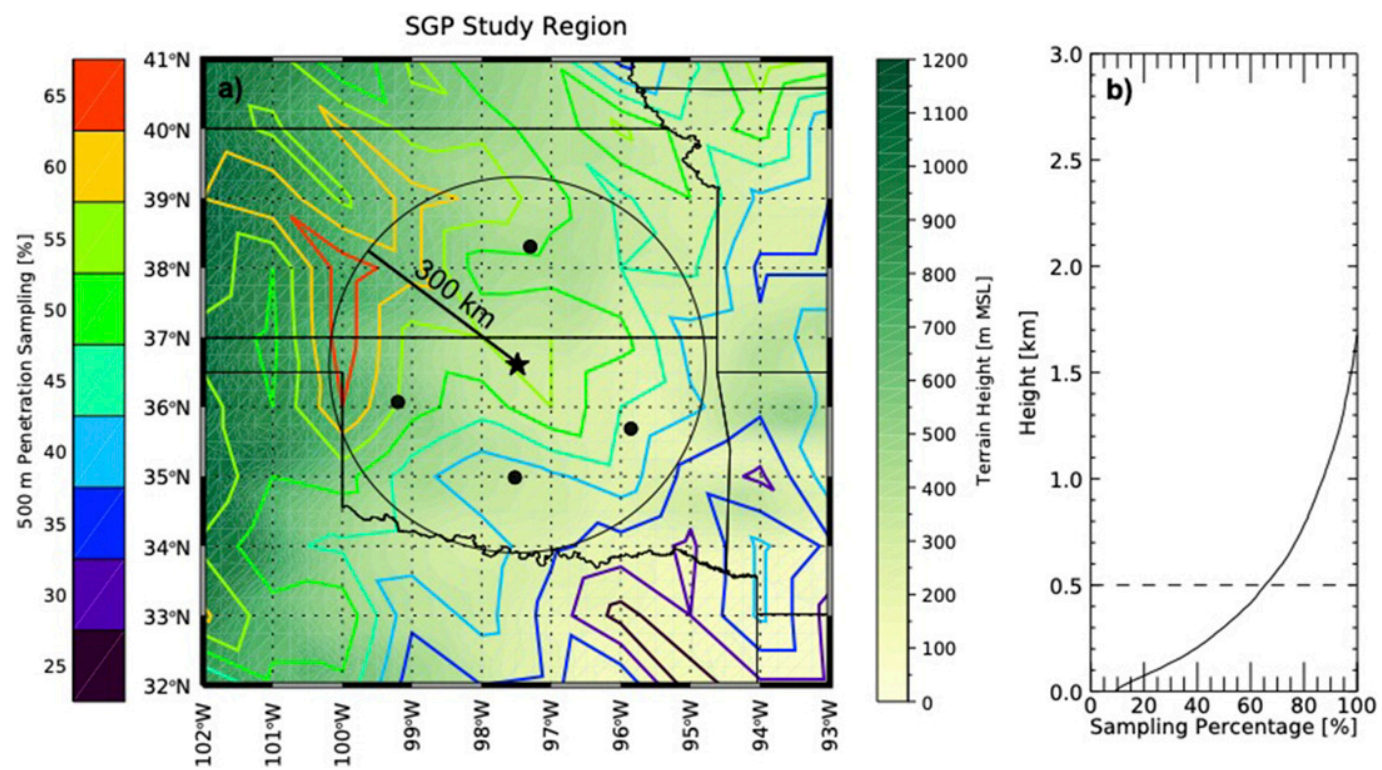

FIG. 1. (a) Terrain height (color-filled contours) of the study region from ERA5 over the southern Great Plains along with the fractional sampling of COSMIC RO soundings penetrating to within $500 \mathrm{~m}$ above the surface (colored contour lines). The ARM central (C1) station (black star) and ancillary support stations (black dots) are also shown. The black open circle denotes $300-\mathrm{km}$ collocation radius as labeled. (b) Vertical profile of sampling percentage for all COSMIC RO refractivity profiles as a function of penetration height.

sensing the lowest $100 \mathrm{~m}$ above the surface due to receiver tracking issues and the limited vertical resolution of RO retrievals (Yu et al. 2018). In addition, the gradient method only retrieves one PBLH value for each profile and could be most reliable for convective boundary layers in the presence of one dominant sharp gradient layer. But the gradient method can be sensitive to the vertical sampling interval of the profile, especially in the presence of two or more sharp gradient layers (e.g., residual layers). To alleviate the PBLH detection uncertainty, the $30-\mathrm{m}$ smoothing is applied to radiosonde profiles (section $2 \mathrm{~b}$ ) to remove large gradients caused by very fine structures and allow for more robust and consistent PBLH detection with RO data, which have approximately $100 \mathrm{~m}$ vertical resolution in the lower troposphere.

\section{e. Diurnal variation analysis with multilinear harmonic analysis method}

This study aims to examine the diurnal characteristics of the PBL over the SGP region, specifically the diurnal amplitude of the PBLH. Several more complicated methods (i.e., fast Fourier transform, power spectrum analysis) for finding the diurnal amplitude have been derived. In our study, however, we use the simple statistical methods that can approximate the results of more complicated analyses and provide similar results as Dai et al. (2002) and Xie et al. (2010) as briefly described below.

Given a time series of PBLH observations $z_{i}, i=1,2, \ldots$, $M$, where $M$ is the maximum number of samples for a day (e.g., $M=4$ for 6-hourly radiosondes), as a function of local solar time $t_{i}$, it can be decomposed into three components:

$$
z_{i}\left(t_{i}\right)=\bar{z}+\sum_{i=1}^{M} S_{M}\left(t_{i}\right)+\epsilon,
$$

where $\bar{z}$ is the daily mean PBLH, $S_{M}$ represents the 24- and 12-h harmonics, and $\epsilon$ is the residual noise. The harmonics of different time scales can be expressed as the linear combination of cosine and sine waves with a given amplitude and phase such that

$$
S_{M}=A_{M} \cos \left(M t^{\prime}-\phi\right)=a_{M} \cos \left(M t^{\prime}\right)+b_{M} \sin \left(M t^{\prime}\right),
$$

where $t^{\prime}$ is the local solar time expressed in radians and is equal to $t^{\prime}=\left(2 \pi t_{i}\right) / 24$, where $t_{i}$ is LST in hours; $M=1,2,3$, $\ldots$, denotes the harmonic with a period of $24,12,8, \ldots$, hours, respectively; $A_{M}$ is the one-sided amplitude of the wave; and $\phi$ is the phase (or time of the maximum $z_{i}$ in LST. In this study, we focus only on the diurnal (24-h, $S_{1}$ ) component. A similar harmonic analysis done for the radiosondes is also done using the collocated RO profiles. Because the RO profiles have significantly higher temporal resolution than the radiosondes, we bin the RO profiles into eight LST bins. Each bin is $3 \mathrm{~h}$ wide, from 0000 to 2100 LST centered on the bin with $\pm 1.5 \mathrm{~h}$ on either side. Constructing the bins in this way prevents double counting of RO profiles such that each RO sounding will be uniquely assigned to its corresponding bin. It is worth noting that the 3-hourly bin was chosen after testing different bin sizes aiming to find the most uniform sampling for each bin. Each 3-h bin contains an average of about $150 \mathrm{RO}$ profiles. Whereas for hourly 

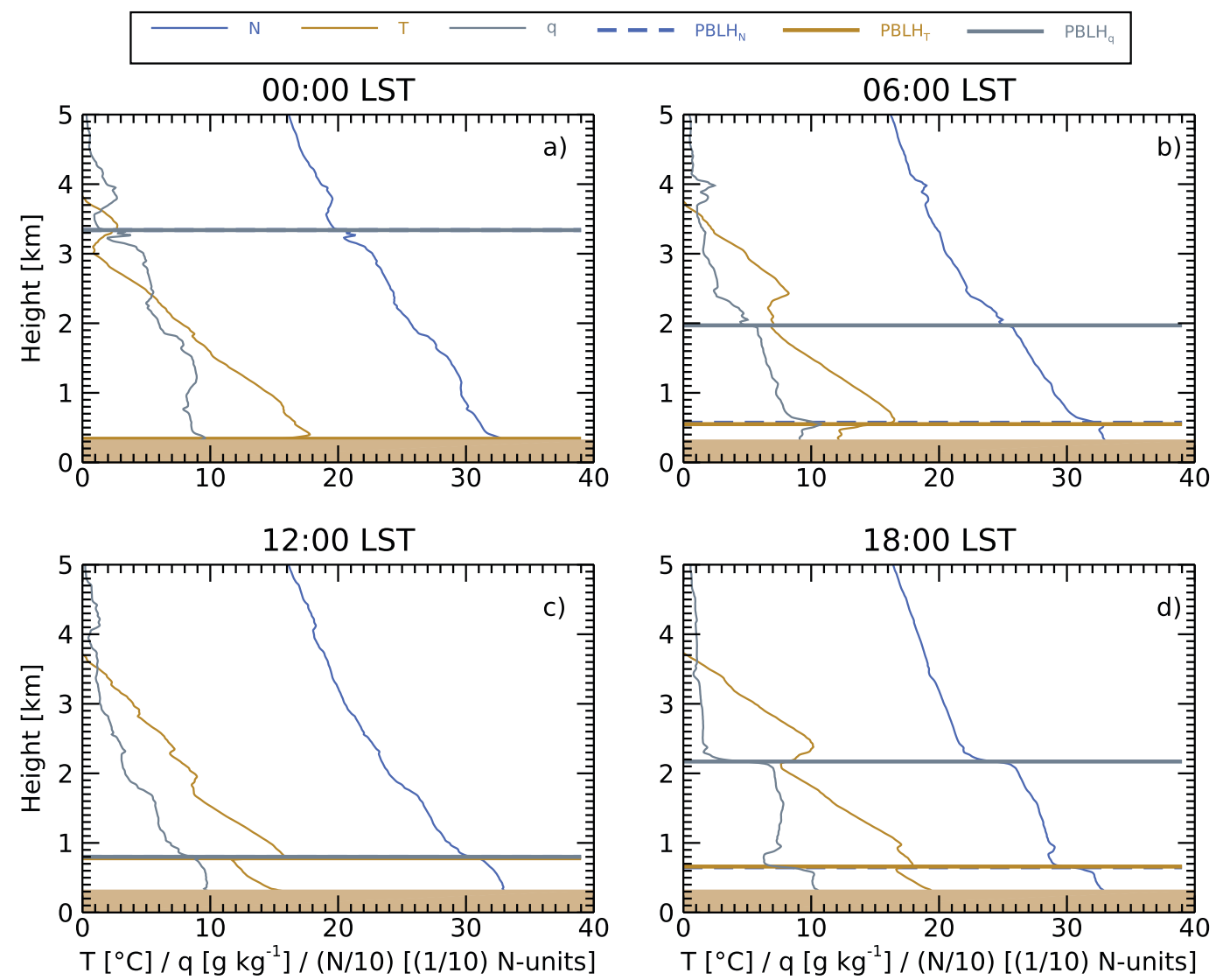

FIG. 2. Typical radiosonde profiles of temperature $\left({ }^{\circ} \mathrm{C}\right.$, gold), specific humidity $\left(\mathrm{g} \mathrm{kg}^{-1}\right.$, gray), and refractivity (N-units/10, blue) from the SGP C1 station at four local times (0000, 0600, 1200, 1800 LST) on 14 Mar 2007 above MSL. The local terrain height (approximately $300 \mathrm{~m}$ ) marked by tan bars in each panel.

bins, the minimum bin sampling was 44 , and the maximum bin sampling was 73 (not shown).

Note that the PBLH diurnal amplitude is generally an order smaller than the PBLH value. Therefore, we extend the diurnal analysis to PBLH anomalies, i.e., PBLH of individual profile subtracting the mean PBLH at each synoptic time bin. The amplitude and phase of the PBLH anomalies can then be derived.

\section{PBL characteristics from radiosonde over SGP}

\section{a. Diurnal variation in PBL vertical structure}

Figure 2 shows 6-hourly radiosonde soundings from 14 March 2007 at each launch time at the $\mathrm{C} 1$ station. The profiles in Fig. 2 were chosen for aesthetic reasons because they show common features seen in profiles over the course of the study, as well as illustrate the relationship between the gradient method of determining the PBLH. Note the regular UTC time is converted to LST in the central time zone, United States, by (UTC $-6 \mathrm{~h}$ ). It is easy to see that the shallow surface temperature inversions (typically between 100 and $300 \mathrm{~m}$ ) occur in the 0000 and 0600 LST vertical profiles, and the temperature-derived $\mathrm{PBLH}_{T}$ sits close to the height of the inversion base, where the maximum gradient in the profile occurs as indicated by horizontal lines in Fig. 2. The $\mathrm{PBLH}_{T}$ tends to be lower during the midnight and early morning hours (0000 and 0600 LST) and rises throughout the daylight hours coincident with solar heating. The specific humidity profiles $(q)$ begin to stratify during the daytime, when sharper gradients tend to appear between 1200 and 1800 LST. The refractivity $(N)$ is a function of both temperature and moisture content, and thus, the $\mathrm{PBLH}_{N}$ exhibits the characteristics of both temperature and moisture. For example, during the overnight hours, the $\mathrm{PBLH}_{N}$ coincides with $\mathrm{PBLH}_{T}$, while during the day, the $\mathrm{PBLH}_{N}$ matches better with the $\mathrm{PBLH}_{q}$.

One important characteristic that is seen regularly in the radiosonde profiles from the SGP is a multilayer vertical structure in both the temperature and specific humidity profiles. As described in section 2d, the PBLH detection methodology only retrieves one PBLH value corresponding to the strongest gradient for each profile and could be sensitive to the vertical sampling interval of the profile. However, in the presence of multilayer structures presented in the high-resolution radiosonde profiles (Fig. 2), the algorithm may lead to different PBLH from different variables for the same profile (e.g., Seidel et al. 2010). This characteristic is, however, outside the scope of the paper and merits further study in the future. 

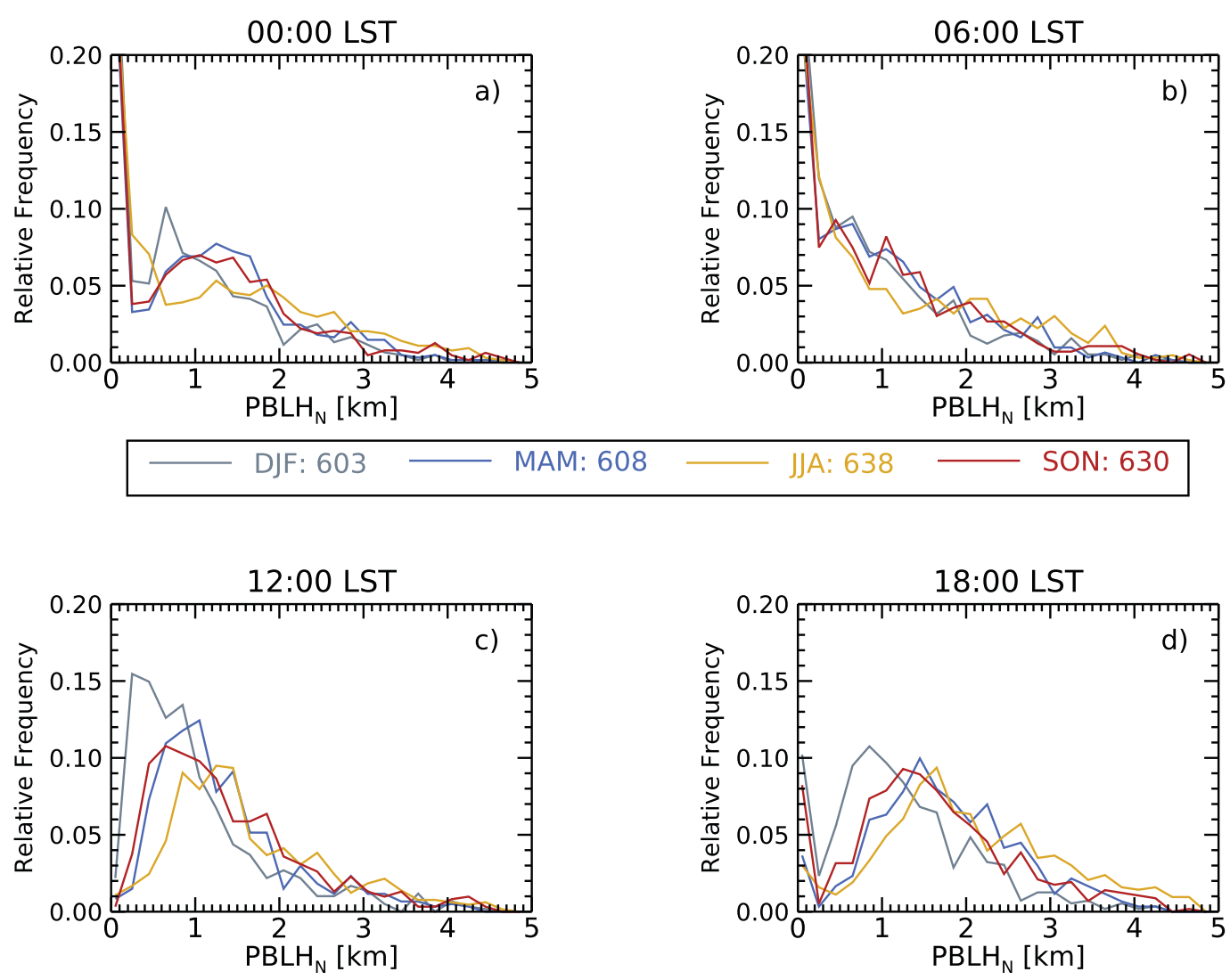

FIG. 3. Histograms of radiosondes $\mathrm{PBLH}_{N}$ in four seasons (DJF, gray; MAM, blue; JJA, gold; and SON, red) at four local synoptic times. The total number of samples for each season is indicated in the legend.

\section{b. Histograms of PBLH from radiosonde soundings}

Based on the analysis of the 7 years of radiosonde data from $\mathrm{C} 1$ station and four ancillary SGP stations (seen in Fig. 1), the PBLH values were evenly binned from 0 to $5 \mathrm{~km}$ at intervals of $100 \mathrm{~m}$. Figure 3 shows the relative frequency histograms of radiosonde refractivity-derived $\mathrm{PBLH}_{N}$ at each local solar time and subset by season. The frequency values are plotted at the center of each bin.

The $\mathrm{PBLH}_{N}$ during the overnight and early morning at 0000 and 0600 LST both show a dominant frequency peak at very shallow PBLH $(\sim 100 \mathrm{~m})$. A secondary peak around $1000 \mathrm{~m}$ is clearly seen at 0000 LST and less evident at 0600 LST. However, much deeper PBLH (approximately $1 \mathrm{~km}$ ) are observed during the daytime at 1200 and 1800 LST. In addition, distinctions between winter (DJF) and summer (JJA) at all LST are observed. For example, the $\mathrm{PBLH}_{N}$ tends to peak at lower heights in winter than those in summer, and the spring (MAM) and autumn (SON) seasons fall in between. Moreover, the daytime PBLH peaks between 500 and $1000 \mathrm{~m}$ depending on the season at 1200 LST and between 1000 and $1500 \mathrm{~m}$ around 1800 LST. Note that refractivity is a constituent variable that depends on pressure, temperature, and moisture, and thus, the histogram of
$\mathrm{PBLH}_{N}$ could be similar to, but distinctly different from, $\mathrm{PBLH}_{T}$ and $\mathrm{PBLH}_{q}$ histograms.

Similar to the histogram pattern of $\mathrm{PBLH}_{N}$, the $\mathrm{PBLH}_{T}$ histograms shown in Fig. 4 also show a dominant peak at very shallow heights ( $\sim 100 \mathrm{~m}$, i.e., surface inversion height) overnight and in the early morning hours, and transition to a more normal distribution during the daytime and evening hours. Also, the $\mathrm{PBLH}_{T}$ tends to peak at lower heights in DJF than those in JJA. However, the overall distribution of $\mathrm{PBLH}_{T}$ tends to slightly shift to shallower heights with reduced width as compared to $\mathrm{PBLH}_{N}$. Moreover, in JJA, the shallow $\mathrm{PBLH}_{T}(<1000 \mathrm{~m})$ observations during 0000-0600 LST make up a disproportionately large fraction and, as a result, few $\mathrm{PBLH}_{T}$ over $1000 \mathrm{~m}$ are observed.

Figure 5 shows the histograms of specific humidity-derived PBLH $\left(\mathrm{PBLH}_{q}\right)$, which are very similar to those of $\mathrm{PBLH}_{N}$ and $\mathrm{PBLH}_{T}$ across all times and seasons. Again, the results show MAM and SON acting as transition seasons and having characteristics of both DJF and JJA. However, major differences are seen in the less pronounced peak at shallow $\mathrm{PBLH}_{q}$ $(\sim 100-200 \mathrm{~m})$ and increased frequency of higher $\mathrm{PBLH}_{q}$, especially at 0000 and 0600 LST, which was not seen in $\mathrm{PBLH}_{N}$ and $\mathrm{PBLH}_{T}$. A wider spread in the $\mathrm{PBLH}_{q}$ 

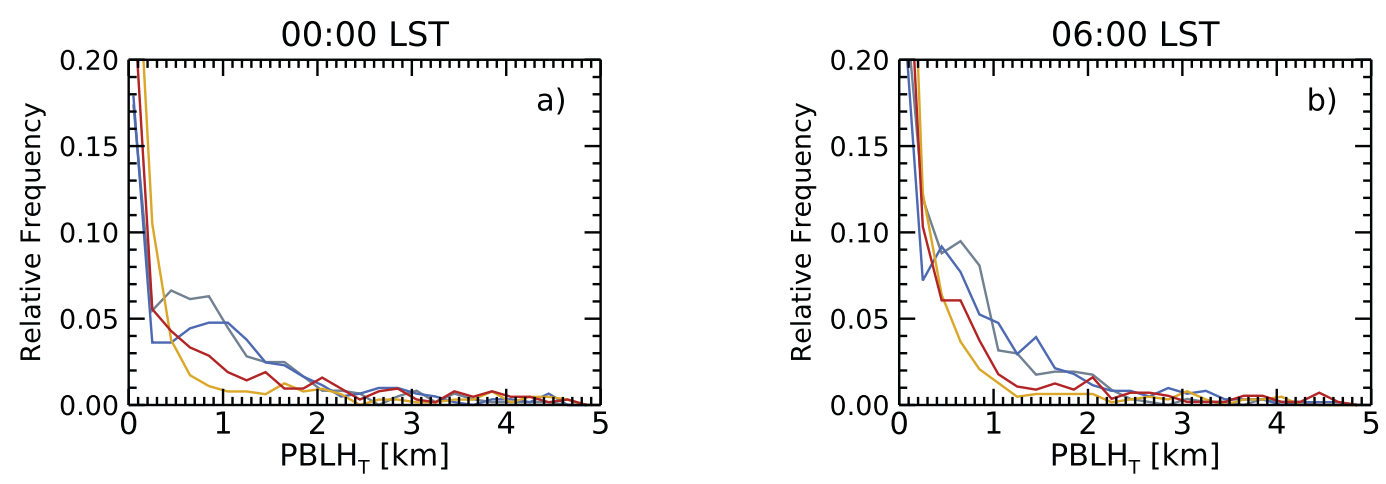

- DJF: $603-$ MAM: $608-$ JJA: $638-$ SON: 630
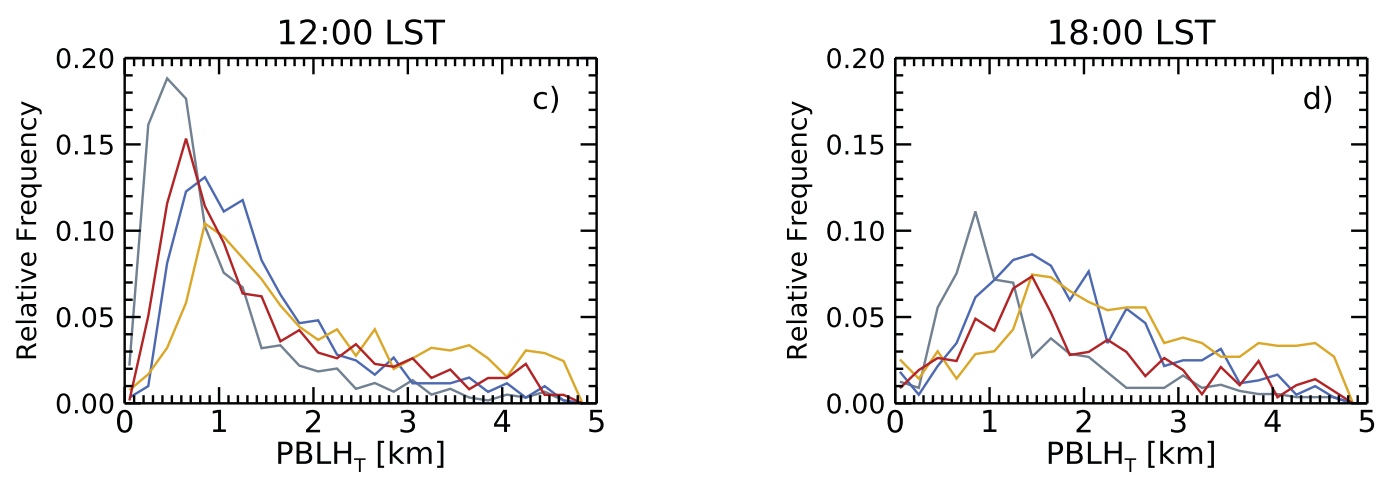

FIG. 4. Histograms of radiosonde $\mathrm{PBLH}_{T}$ in four seasons (DJF, gray; MAM, blue; JJA, gold; and SON, red) at four local synoptic times. The total number of samples for each season is indicated in the legend.

observations indicates the minimum specific humidity gradient could be sensitive to the fine structures in the specific humidity profile when no dominant humidity gradient is present.

\section{c. Diurnal variation of $P B L H$ from radiosondes}

The diurnal amplitude and phase of changes in the boundary layer height over the SGP can have significant implications for energetics in the lower layers of the atmosphere. After binning all 7 years of radiosonde profiles into four LST bins (0000, 0600, 1200, 1800 LST) corresponding to radiosonde launch times, the mean PBLH anomalies are shown in Fig. 6. Standard errors around the mean value are shown using error bars. Furthermore, the multilinear harmonic analysis (section 2e) is carried out, and the diurnal component of the PBLH anomalies from each variable is shown as a smooth sinusoidal curve.

$\mathrm{PBLH}_{T}$ shows the largest diurnal amplitude of about 600 $\mathrm{m}$, peaking around $1500 \mathrm{LST}$, coincident with the maximum local solar heating. The diurnal amplitude of $\mathrm{PBLH}_{q}$ is much smaller $(\sim 300 \mathrm{~m})$ and peaks around midnight. Interestingly, the diurnal amplitude of $\operatorname{PBLH}_{N}(\sim 230 \mathrm{~m})$ is more consistent with $\mathrm{PBLH}_{q}$ but is more in phase with $\mathrm{PBLH}_{T}$ peaking at $\sim 1700$ LST, shortly after that of the $\mathrm{PBLH}_{T}$. The $\mathrm{PBLH}_{T}$ was found to have the largest diurnal amplitude $(691 \mathrm{~m})$ peaking at 1545 LST. $\operatorname{PBLH}_{q}$ had the second highest amplitude (278 m) peaking overnight just after 2300 LST. Whereas the $\mathrm{PBLH}_{N}$ exhibits the smallest amplitude $(232 \mathrm{~m})$ peaking at 1730 LST.

\section{PBL characteristics from COSMIC RO over the SGP}

The quasi-random spatial and local time sampling of the high-vertical-resolution COSMIC RO observations enables a better method for PBL diurnal analysis. To allow direct comparison between the COSMIC RO and radiosonde diurnal analysis, the COSMIC RO soundings within $3 \mathrm{~h}$ and 300 $\mathrm{km}$ of the SGP C1 station (and 4 ancillary stations) during the 7-yr period (2007-13) were identified. Note that only COSMIC refractivity profiles that penetrate below $500 \mathrm{~m}$ above local terrain height were included. A total of 1335 pairs of radiosondes and collocated COSMIC RO soundings were found.

\section{a. Histograms of PBLH $H_{N}$ from COSMIC RO and collocated radiosondes}

Similar to the radiosonde analysis in section 3a, the $\mathrm{PBLH}_{N}$ from COSMIC RO refractivity profiles were evenly binned 

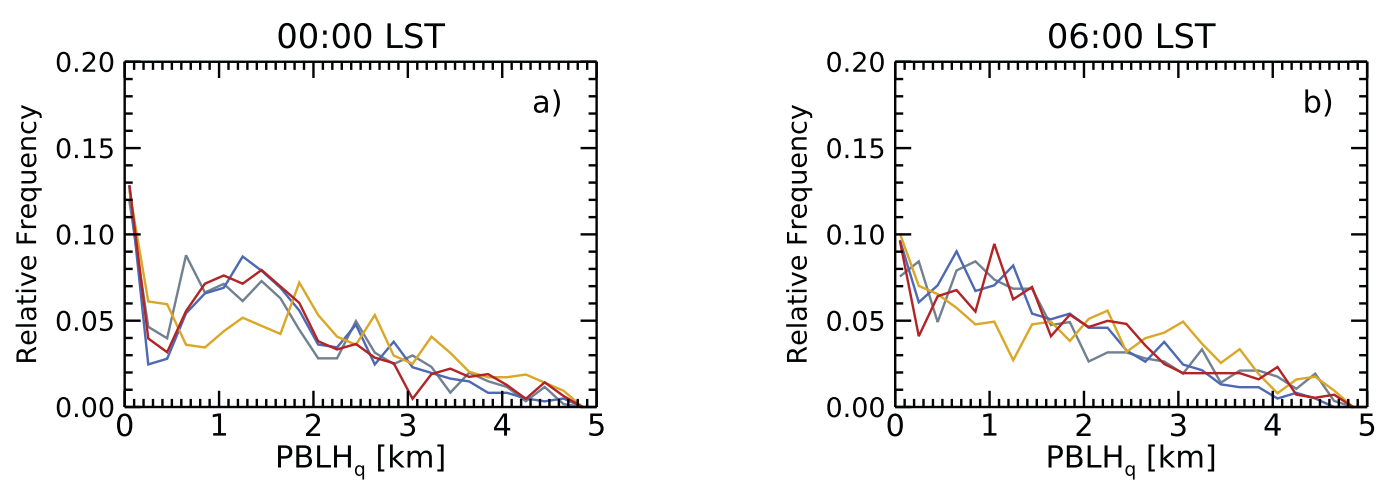

\begin{tabular}{|llll}
- DJF: $603-$ MAM: $608-$ JJA: $638-$ SON: 630 \\
\hline
\end{tabular}
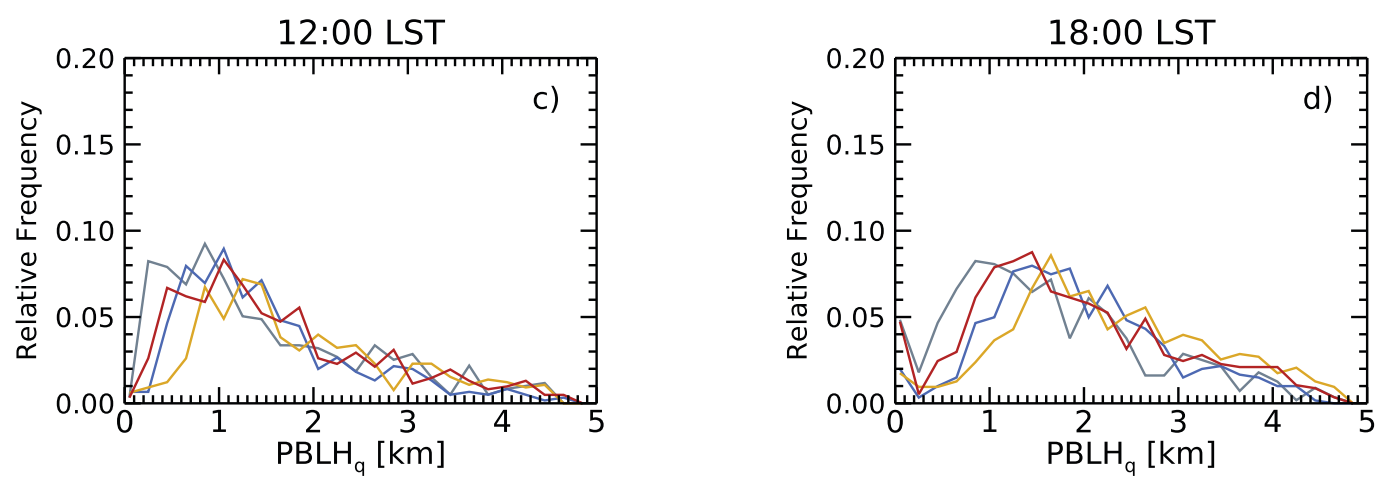

FIG. 5. Histograms of radiosonde $\mathrm{PBLH}_{q}$ in four seasons (DJF, gray; MAM, blue; JJA, gold; and SON, red) at four local synoptic times. The total number of samples for each season is indicated in the legend.

with 100-m intervals from 0 to $5 \mathrm{~km}$. Figure 7 shows the histograms of $\mathrm{PBLH}_{N}$ at four local solar times from the radiosondes and the collocated COSMIC RO observations. First, it

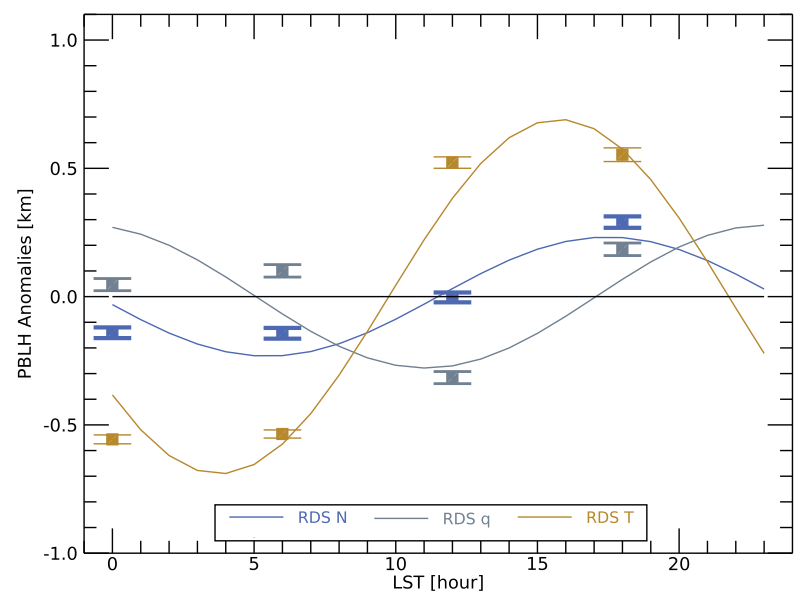

FIG. 6. Diurnal variation of the mean PBLH anomalies based on radiosonde temperature (gold squares), specific humidity (gray squares), and refractivity observations (blue squares) and the corresponding standard error (error bars) at four local solar times. The least squares diurnal fit of the PBLH anomaly for each variable are plotted as solid lines with their respective colors. is important to note that the histogram of the $\mathrm{PBLH}_{N}$ from the radiosonde subset is consistent with the histogram for all 7 years of radiosondes as shown in Fig. 3, indicating the down sampling still preserves the key features of the PBLH. Overall, good agreement in $\mathrm{PBLH}_{N}$ between the COSMIC RO and the collocated radiosondes is seen at all LSTs, especially during the 1200 and 1800 LST hours. However, the shallow PBLH ( 100-200 m) most frequently seen during the night time (0000-0600 LST) in radiosonde observation is rarely observed in COSMIC RO observations. This is somewhat expected as it is challenging for RO to detect the surface inversion due to the limited vertical resolution of RO observations (100-200 m). In addition, RO observations represent an along-path average, while radiosonde observations are point-like in situ profile measurements. Therefore, RO could miss the capture of localized surface inversion events due to the inhomogeneity along the RO ray path as a result of uneven topography and surface heating.

It is worth noting, however, that very shallow RO-derived PBLH values should be considered with some additional caution, because many profiles that showed extremely low PBLH values (e.g., $<200 \mathrm{~m}$ ) identified the PBLH as the last point in the profile or within $200 \mathrm{~m}$ of the last point in the profile. Furthermore, when considering PBLH values lower than $500 \mathrm{~m}$, the possibility of sampling bias increases, particularly 

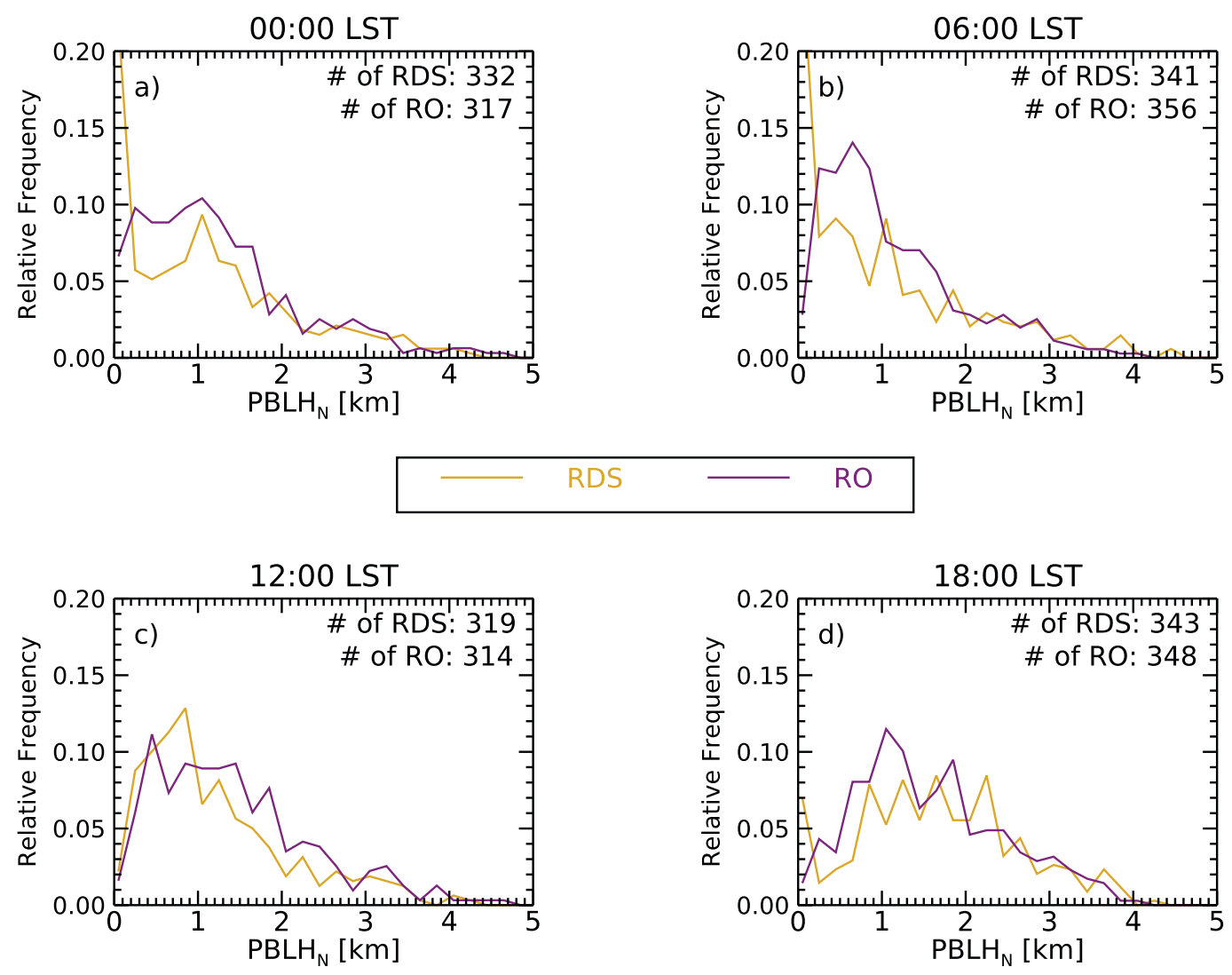

FIG. 7. Histograms of $\mathrm{PBLH}_{N}$ for collocated COSMIC RO (purple) and radiosondes (RDS, gold) at four local solar times.

for PBLH values less than $100 \mathrm{~m}$, simply due to RO penetration (see Fig. 1b). Despite this, there is still some value in examining the evolution of the PBLH as defined here since it can capture some aspects of PBL diurnal changes (e.g., residual layer) over the course of the day.

\section{b. Diurnal variation of $P B L H_{N}$ from COSMIC RO and collocated radiosondes}

COSMIC RO profiles are quasi random in both space and local time and thus could offer more details in the characteristics of PBL diurnal variation as compared to the radiosondes with only four local time observations. In this study, only the $\mathrm{PBLH}_{N}$ derived from RO refractivity profiles were compared to radiosondes, as the temperature and humidity retrieval from RO are not independent observations and embed model a priori uncertainty. Similar to section $3 \mathrm{c}$, the radiosonde $\mathrm{PBLH}_{N}$ data are binned into four 6-h bins centered on 0000, 0600, 1200, and 1800 LST, then the PBLH anomalies and standard error at each of the four LST are derived. On the other hand, the collocated COSMIC RO $\mathrm{PBLH}_{N}$ data are binned into eight 3-h LST bins, centered on four radiosonde times, plus four additional times (0300, 0900, 1500, 2100 LST). Each COSMIC RO 3-h data bin contains between 146 and 187 RO profiles. The diurnal component of the PBLH anomalies is then derived using the multilinear harmonic analysis (section 2e).

Figure 8 shows the mean $\mathrm{PBLH}_{N}$ anomalies (squares) and standard error (error bars) for both COSMIC RO and collocated radiosondes. The mean $\mathrm{PBLH}_{N}$ anomalies from the radiosondes in Fig. 8 (gold) are the same anomalies shown in Fig. 6 at 0000, 0600, 1200, and 1800 LST, where shallower $\mathrm{PBLH}_{N}$ values are seen overnight, and deeper $\mathrm{PBLH}_{N}$ during the daytime. The RO-derived $\mathrm{PBLH}_{N}$ (purple) matches the radiosonde-derived $\mathrm{PBLH}_{N}$ quite well, indicating that COSMIC RO captures the diurnal variation of the terrestrial $\mathrm{PBLH}_{N}$.

We note that the diurnal amplitude of the $\mathrm{PBLH}_{N}$ from radiosondes is $232 \mathrm{~m}$, while the COSMIC RO $\mathrm{PBLH}_{N}$ amplitude is $283 \mathrm{~m}$. The standard errors of the RO observations have similar magnitude of approximately 100-200 m compared to the radiosondes' $100 \mathrm{~m}$. Additionally, the $\mathrm{PBLH}_{N}$ peaks at 1637 LST for the RO and about one hour later at 1749 LST for the radiosonde. Given the results of the diurnal analysis, it is clear that RO refractivity profile observations are capable of resolving the diurnal variation of the PBLH by showing similar phase, and amplitude as the collocated radiosondes. A summary of the amplitudes and phases from the harmonic analysis of all of the radiosonde and RO PBLH can be found in Table 1. 


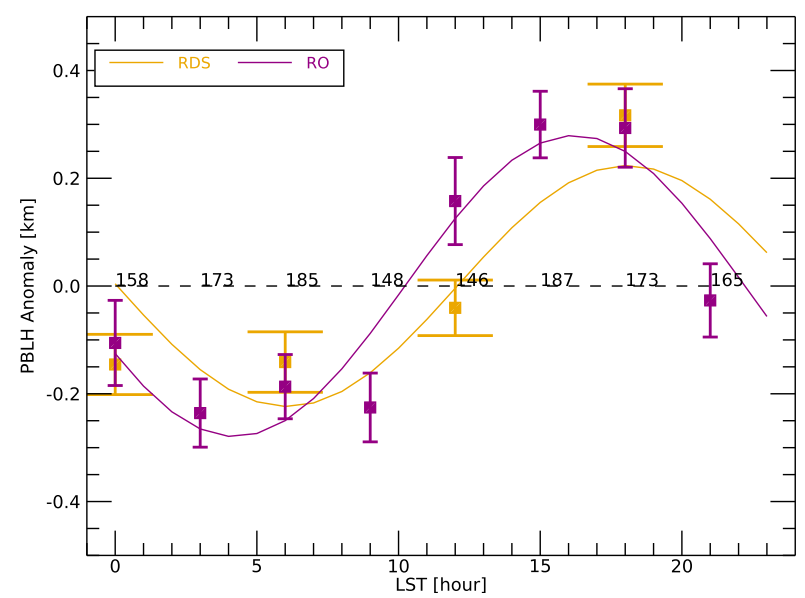

FIG. 8. Diurnal variation of the mean $\mathrm{PBLH}_{N}$ anomalies from radiosonde (gold squares) and COSMIC RO (purple squares) with the corresponding standard error represented by error bars. The least squares diurnal fit of the $\mathrm{PBLH}_{N}$ anomaly is represented by the solid lines. Numbers along the $x$ axis indicate the number of samples in each RO bin.

In an attempt to better understand the seasonal variability of the PBLH amplitudes and phases, the data in Fig. 8 were subset by season. Figure 9 shows the mean $\mathrm{PBLH}_{N}$ anomalies (squares) and their standard errors (error bars) from collocated COSMIC RO and radiosonde profiles in each season, separately. Each panel also shows the diurnal component of the harmonic analysis (section 2e), similar to Fig. 8. In general, the differences between radiosonde and RO mean $\mathrm{PBLH}_{N}$ values are slightly larger than those in Fig. 8, but such differences are likely due to decreased sampling in the seasonal subsets. The diurnal bin sampling for COSMIC RO across the seasons remains mostly the same due to the quasirandom sampling nature of RO.

However, it is clear that there is some amount of seasonal variability in the $\mathrm{PBLH}_{N}$ diurnal amplitudes and phases. During DJF, the diurnal variations of $\mathrm{PBLH}_{N}$ for COSMIC RO and radiosondes overlap with each other with identical amplitude and phase within an hour of each other, which is consistent with the annual mean diurnal phases and amplitudes seen in Table 1 and Fig. 8. During MAM, the diurnal amplitude of the $\mathrm{PBLH}_{N}$ increases to around $400 \mathrm{~m}$ for RO but remains close to the DJF value of just over $200 \mathrm{~m}$ for radiosondes. In addition, the RO $\mathrm{PBLH}_{N}$ peaks slightly earlier at around 1600 LST rather than closer to 1700 LST for radiosonde also seen during DJF. During JJA, the $\mathrm{PBLH}_{N}$ from both the RO and

TABLE 1. Diurnal amplitude and phase for radiosonde and collocated COSMIC RO.

\begin{tabular}{lcc}
\hline \hline \multicolumn{1}{c}{ Diurnal (24 h) } & Amplitude $(\mathrm{km})$ & Phase (LST) \\
\hline Radiosonde PBLH $_{q}$ & 0.278 & 2303 \\
Radiosonde PBLH $_{T}$ & 0.691 & 1545 \\
Radiosonde PBLH $_{N}$ & 0.232 & 1729 \\
COSMIC RO PBLH $_{N}$ & 0.283 & 1622 \\
\hline
\end{tabular}

the radiosonde is in phase and peaks at 1530 LST, likely due to the influence of local solar heating. The amplitude of the $\mathrm{PBLH}_{N}$ from the RO also decreases from that of MAM to about $300 \mathrm{~m}$. Finally, during SON, RO and radiosonde show similar diurnal amplitudes of $\mathrm{PBLH}_{N}$, but are almost $3 \mathrm{~h}$ out of phase with each other. The seasonal differences in diurnal PBLH variations are likely due simply to seasonal changes in temperature. However, the transitional seasons (MAM and SON) are likely to incorporate characteristics of both surrounding seasons, and possibly leads to the variability and differences in diurnal phase and amplitude (Fig. 9).

\section{Conclusions and future work}

In this study, we have analyzed 7 years of radiosonde soundings and COSMIC RO profiles over the SGP. The PBL diurnal characteristics from radiosonde soundings over the Southern Great Plains were shown. The simple gradient method for PBLH detection was implemented on RO refractivity as well as radiosonde temperature, specific humidity, and refractivity profiles. It is worth noting that that the gradient method for $\mathrm{PBLH}_{q}$ could encounter difficulties identifying the PBLH in the presence of very shallow surface inversion around midnight.

There is clear diurnal variation in the PBLH, particularly for temperature derived PBLH $\left(\mathrm{PBLH}_{T}\right)$ that shows shallow surface inversion overnight, but much deeper during the daytime due to local solar heating. Such features, however, are not seen in $\mathrm{PBLH}_{q}$. Since refractivity is a function of temperature and moisture, the $\mathrm{PBLH}_{N}$ histograms show a combination of the distributions of $\mathrm{PBLH}_{T}$ and $\mathrm{PBLH}_{q}$. The PBLH histograms also show clear seasonal variations, with a deeper PBL seen in JJA, shallower PBL in DJF, and a combination of both features in MAM and SON.

Harmonic analysis on all seven years of radiosonde soundings shows that the diurnal component of the $\mathrm{PBLH}_{T}$ and $\mathrm{PBLH}_{N}$ peak in the afternoon between 1500 and 1730 LST at with diurnal amplitude of 691 and $232 \mathrm{~m}$, respectively. On the other hand, the $\mathrm{PBLH}_{q}$ peaks overnight just before midnight at $287 \mathrm{~m}$. The standard error for all of the radiosonde PBLH values is approximately $100 \mathrm{~m}$, likely due to seasonal- and synoptic-scale influences.

Directly comparing the $\mathrm{PBLH}_{N}$ derived from collocated COSMIC RO and radiosonde refractivity profiles shows extremely good agreement in both the diurnal amplitude and phase, strongly indicating RO's capability to resolve PBL diurnal variations over land. $\mathrm{RO}$-derived $\mathrm{PBLH}_{N}$ peaks about 1-2 $\mathrm{h}$ ahead of the radiosonde-derived $\mathrm{PBLH}_{N}$ with a difference in amplitude of merely $50 \mathrm{~m}$.

It appears that RO is able to successfully detect upward of $50 \%$ of shallow PBLH below $500 \mathrm{~m}$ as indicated by the collocated radiosondes. However, it can be very difficult for RO to accurately detect very shallow surface inversions (especially $<200 \mathrm{~m}$ ) commonly seen overnight (Figs. 3-5) with RO due to the limited vertical resolution (approximately 100-200 m) and the inherent limb sounding geometry of the RO profile. In addition, the simple gradient method to identify the PBLH 

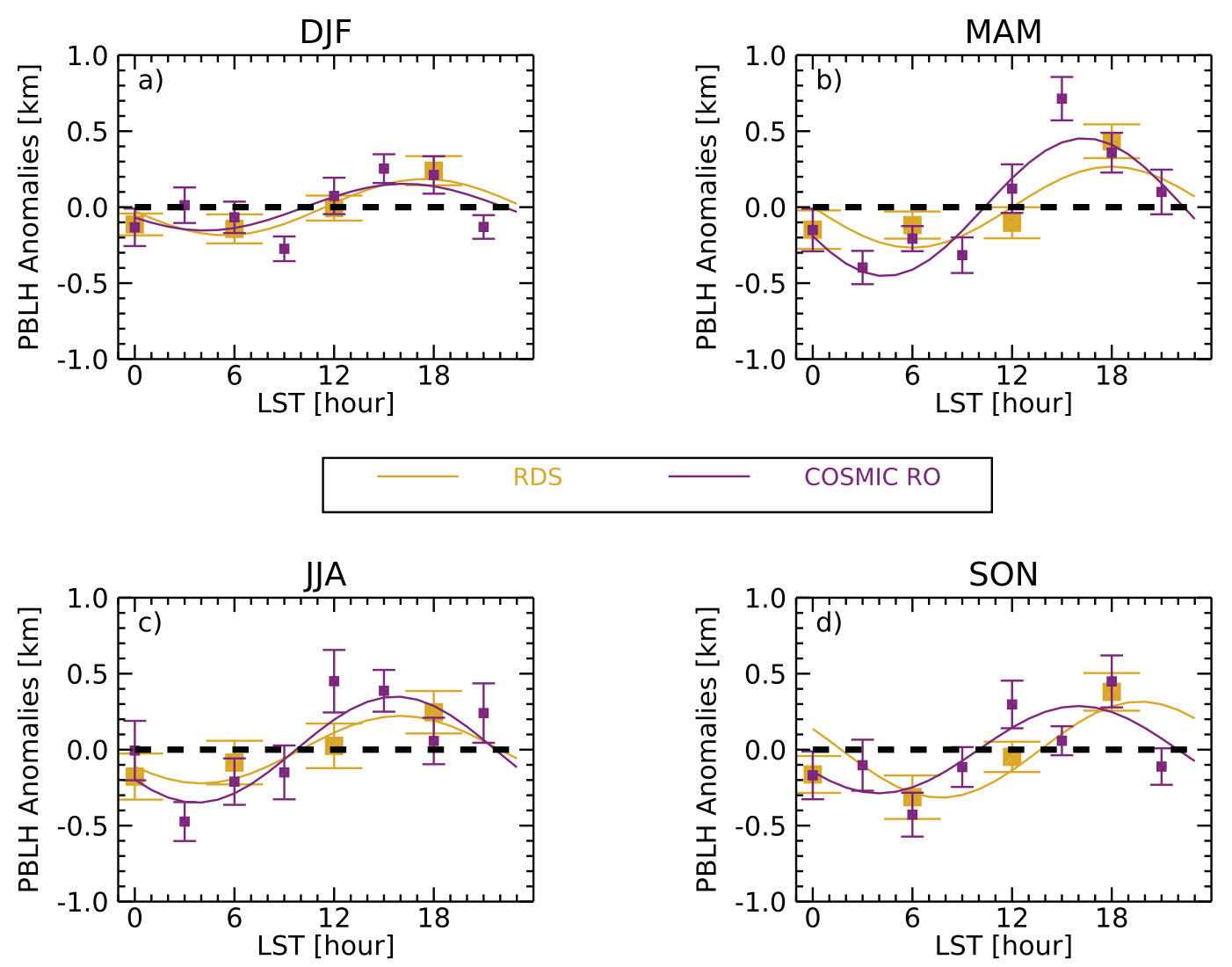

FIG. 9. Seasonal mean of $\mathrm{PBLH}_{N}$ diurnal variations from radiosonde (gold squares) and GNSS RO (purple squares) with the corresponding standard error represented by error bars. The least squares diurnal fit of the $\mathrm{PBLH}_{N}$ anomaly is represented by the solid lines.

in this study could encounter challenges when multiple-layer structures with similar gradients that are commonly seen over the SGP (e.g., Fig. 2). A more robust PBLH detection algorithm warrants further study.

With the successful launch of the COSMIC-2 mission in June 2019 (Ho et al. 2020), along with recent commercial RO missions (e.g., SPIRE, GeoOptics, and Planet-iQ) and other airborne RO platforms, significant increases in GNSS RO observations in both spatial and temporal space have been achieved and continue to improve. In addition, the higher gain antennas and the newer generation GNSS RO receivers on board the COSMIC-2 LEO satellites enable higher-quality PBL sounding with deeper penetration. Such high-quality GNSS RO PBL soundings will vastly benefit the global PBL diurnal analysis and the PBL physical process study in the future.

Acknowledgments. The authors acknowledge funding support from NASA Grant NNH14ZDA001N-GNSS in completion of this work. Authors K. Nelson and F. Xie are also partially supported by NASA Grant NNX15AQ17G. Work performed by C. Ao and M. Oyola was carried out at the Jet Propulsion Laboratory, California Institute of Technology, under a contract with the National Aeronautics and Space Administration.
Data availability statement. The GNSS RO retrievals are provided by UCAR COSMIC Data Analysis and Archive Center (CDAAC). The radiosonde data were provided by the Atmospheric Radiation Measurement (ARM) program and retrieved using the ARM Data Discovery tool.

\section{REFERENCES}

Albrecht, B. A., C. S. Bretherton, D. Johnson, W. H. Scubert, and A. S. Frisch, 1995: The Atlantic Stratocumulus Transition Experiment-ASTEX. Bull. Amer. Meteor. Soc., 76, 889-904, https://doi.org/10.1175/1520-0477(1995)076<0889: TASTE $>2.0 . \mathrm{CO} ; 2$.

Anthes, R. A., and Coauthors, 2008: The COSMIC/FORMOSAT-3 mission: Early results. Bull. Amer. Meteor. Soc., 89, 313-334, https://doi.org/10.1175/BAMS-89-3-313.

Ao, C. O., T. K. Chan, B. A. Iijima, J.-L. Li, A. J. Mannucci, J. Teixeira, B. Tian, and D. E. Waliser, 2008: Planetary boundary layer information from GPS radio occultation measurements. GRAS SAF Workshop on Applications of GPSRO Measurements, Reading, United Kingdom, ECMWF and EUMETSAT, 123-131.

— , G. A. Hajj, T. K. Meehan, D. Dong, B. A. Iijima, A. J. Mannucci, and E. R. Kursinski, 2009: Rising and setting GPS 
occultations by use of open-loop tracking. J. Geophys. Res., 114, D04101, https://doi.org/10.1029/2008JD010483.

—, D. E. Waliser, S. K. Chan, J.-L. Li, B. Tian, F. Xie, and A. J. Mannucci, 2012: Planetary boundary layer heights from GPS radio occultation refractivity and humidity profiles. J. Geophys. Res., 117, D16117, https://doi.org/10. 1029/2012JD017598.

Basha, G., and M. V. Ratnam, 2009: Identification of atmospheric boundary layer height over a tropical station using high-resolution radiosonde refractivity profiles: Comparison with GPS radio occultation measurements. J. Geophys. Res., 114, D16101, https://doi.org/10.1029/2008JD011692.

Dai, A., J. Wang, R. H. Ware, and T. Van Hove, 2002: Diurnal variation in water vapor over North America and its implications for sampling errors in radiosonde humidity. J. Geophys. Res., 107, 4090, https://doi.org/10.1029/ 2001JD000642.

Ganeshan, M., and D. L. Wu, 2015: An investigation of the Arctic inversion using COSMIC RO observations. J. Geophys. Res. Atmos., 120, 9338-9351, https://doi.org/10.1002/ 2015JD023058.

Garratt, J. R., 1994: The atmospheric boundary layer. Earth-Sci. Rev., 37, 89-134, https://doi.org/10.1016/0012-8252(94)90026-4.

Guo, P., Y. H. Kuo, S. V. Sokolovskiy, and D. H. Lenschow, 2011: Estimating atmospheric boundary layer depth using COSMIC radio occultation data. J. Atmos. Sci., 68, 17031713, https://doi.org/10.1175/2011JAS3612.1.

Hersbach, H., and Coauthors, 2020: The ERA5 global reanalysis. Quart. J. Roy. Meteor. Soc., 146, 1999-2049, https://doi.org/10. 1002/qj.3803.

Ho, S., L. Peng, R. A. Anthes, Y.-H. Kuo, and H.-C. Lin, 2015: Marine boundary layer heights and their longitudinal, diurnal, and interseasonal variability in the southeastern Pacific using COSMIC, CALIOP, and radiosonde data. J. Climate, 28, 2856-2872, https://doi.org/10.1175/JCLI-D-14-00238.1.

- , and Coauthors, 2020: The COSMIC/FORMOSAT-3 radio occultation mission after 12 years: Accomplishments, remaining challenges, and potential impacts of COSMIC-2. Bull. Amer. Meteor. Soc., 101, E1107-E1136, https://doi.org/10. 1175/BAMS-D-18-0290.1.

Holdridge, D. J., 2020: Balloon-borne sounding system (sonde) instrument handbook. ARM Tech. Rep. DOE/SC-ARM-TR029, 38 pp.

Holtslag, A. A. M., and Coauthors, 2013: Stable atmospheric boundary layers and diurnal cycles: Challenges for weather and climate models. Bull. Amer. Meteor. Soc., 94, 1691-1706, https://doi.org/10.1175/BAMS-D-11-00187.1.

Jordan, N. S., R. M. Hoff, and J. T. Bacmeister, 2010: Validation of Goddard Earth Observing System-version 5 MERRA planetary boundary layer heights using CALIPSO. J. Geophys. Res., 115, D24218, https://doi.org/10. 1029/2009JD013777.

Klein, S. A., and D. L. Hartmann, 1993: The seasonal cycle of low stratiform clouds. J. Climate, 6, 1587-1606, https://doi.org/10. 1175/1520-0442(1993)006<1587:TSCOLS > 2.0.CO;2.

Kral, S. T., and Coauthors, 2018: Innovative Strategies for Observations in the Arctic Atmospheric Boundary Layer (ISOBAR)-The Hailuoto 2017 campaign. Atmosphere, 9, 268, https://doi.org/10.3390/atmos9070268.

Kursinski, E. R., G. A. Hajj, J. T. Schofield, R. P. Linfield, and K. R. Hardy, 1997: Observing Earth's atmosphere with radio occultation measurements using the global positioning system. J. Geophys. Res., 102, 23 429-23 465, https://doi.org/10.1029/97JD01569.
Lee, X., 2018: Fundamentals of Boundary-Layer Meteorology. Springer, 256 pp., https://doi.org/10.1007/978-3-319-60853-2.

Liu, S., and X.-Z. Liang, 2010: Observed diurnal cycle climatology of planetary boundary layer height. J. Climate, 23, 5790-5809, https://doi.org/10.1175/2010JCLI3552.1.

Lundquist, J. K., and Coauthors, 2017: Assessing state-of-the-art capabilities for probing the atmospheric boundary layer: The XPIA field campaign. Bull. Amer. Meteor. Soc., 98, 289-314, https://doi.org/10.1175/BAMS-D-15-00151.1.

Maddy, E. S., and C. D. Barnet, 2008: Vertical resolution estimates in version 5 of AIRS operational retrievals. IEEE Trans. Geosci. Remote Sens., 46, 2375-2384, https://doi.org/10. 1109/TGRS.2008.917498.

Mahrt, L., 1999: Stratified atmospheric boundary layers. Bound.-Layer Meteor., 90, 375-396, https://doi.org/10.1023/ A:1001765727956.

— 2014: Stably stratified atmospheric boundary layers. Annu. Rev. Fluid Mech., 46, 23-45, https://doi.org/10.1146/annurevfluid-010313-141354.

McGibbon, J., and C. S. Bretherton, 2019: Single-column emulation of reanalysis of the northeast Pacific marine boundary layer. Geophys. Res. Lett., 46, 10053-10060, https://doi.org/ 10.1029/2019GL083646.

Mehta, S. K., M. V. Ratnam, S. V. Sunilkumar, D. N. Rao, and B. V. Krishna Murthy, 2017: Diurnal variability of the atmospheric boundary layer height over a tropical station in the Indian monsoon region. Atmos. Chem. Phys., 17, 531-549, https://doi.org/10.5194/acp-17-531-2017.

Nicoll, K., R. G. Harrison, H. Silva, R. Salgado, M. Melgão, and D. Bortoli, 2018: Electrical sensing of the dynamical structure of the planetary boundary layer. Atmos. Res., 202, 81-95, https://doi.org/10.1016/j.atmosres.2017.11.009.

Nuijens, L., and A. P. Siebesma, 2019: Boundary layer clouds and convection over subtropical oceans in our current and in a warmer climate. Curr. Climate Change Rep., 5, 80-94, https:// doi.org/10.1007/s40641-019-00126-x.

Oke, T. R., 1988: Boundary Layer Climates. Routledge, 435 pp.

Palm, S. P., A. Benedetti, and J. Spinhirne, 2005: Validation of ECMWF global forecast model parameters using GLAS atmospheric channel measurements. Geophys. Res. Lett., 32, L22S09, https://doi.org/10.1029/2005GL023535.

Ramanathan, V., R. Cess, E. Harrison, P. Minnis, B. Barkstrom, E. Ahmad, and D. Hartmann, 1989: Cloud-radiative forcing and climate: Results from the Earth Radiation Budget Experiment. Science, 243, 57-63, https://doi.org/10.1126/science.243. 4887.57.

Sawyer, V., and Z. Li, 2013: Detection, variations and intercomparison of the planetary boundary layer depth from radiosonde, lidar and infrared spectrometer. Atmos. Environ., 79, 518-528, https://doi.org/10.1016/j.atmosenv.2013.07.019.

Seidel, D. J., C. O. Ao, and K. Li, 2010: Estimating climatological planetary boundary layer heights from radiosonde observations: Comparison of methods and uncertainty analysis. J. Geophys. Res., 115, D16113, https://doi.org/10.1029/ 2009JD013680.

—, Y. Zhang, A. Beljaars, J.-C. Golaz, A. R. Jacobson, and B. Medeiros, 2012: Climatology of the planetary boundary layer over the continental United States and Europe. J. Geophys. Res., 117, D17106, https://doi.org/10.1029/2012JD018143.

Sisterson, D. L., R. A. Peppler, T. S. Cress, P. J. Lamb, and D. D. Turner, 2016: The ARM Southern Great Plains (SGP) site. The Atmospheric Radiation Measurement (ARM) Program: The 
First 20 Years, Meteor. Monogr., No. 57, Amer. Meteor. Soc., https://doi.org/10.1175/AMSMONOGRAPHS-D-16-0004.1.

Sokolovskiy, S., Y. H. Kuo, C. Rocken, W. S. Schreiner, D. Hunt, and R. A. Anthes, 2006: Monitoring the atmospheric boundary layer by GPS radio occultation signals recorded in the open-loop mode. Geophys. Res. Lett., 33, L12813, https://doi. org/10.1029/2006GL025955.

- , H. Lenschow, Z. Zeng, C. Rocken, S. Schreiner, C. Hunt, Y.-H. Kuo, and A. Anthes, 2011: Monitoring the depth of the atmospheric boundary layer by FORMOSAT-3/COSMIC. Fifth FORMOSAT-3/COSMIC Data Users Workshop, Taipei, Taiwan, NCAR/UCAR, https://opensky.ucar.edu/ islandora/object/conference:925.

Stull, R. B., 1988: An Introduction to Boundary Layer Meteorology. Kluwer Academic Publishers, 666 pp.

—_, E. Santoso, L. K. Berg, and J. Hacker, 1997: Boundary Layer Experiment 1996 (BLX96). Bull. Amer. Meteor. Soc., 78, 1149-1158, https://doi.org/10.1175/1520-0477(1997)078 $<1149$ :BLEB $>2.0 . \mathrm{CO} ; 2$.

Suarez, M. J., A. Arakawa, and D. A. Randall, 1983: The parameterization of the planetary boundary layer in the UCLA general circulation model: Formulation and results. Mon. Wea. Rev., 111, 2224-2243, https://doi.org/10.1175/1520-0493(1983) $111<2224$ :TPOTPB $>2.0$. CO;2.

von Engeln, A., and J. Teixeira, 2013: A planetary boundary layer height climatology derived from ECMWF reanalysis data. $J$. Climate, 26, 6575-6590, https://doi.org/10.1175/JCLI-D-1200385.1 .

Ware, R. H., and Coauthors, 1996: GPS sounding of the atmosphere from low Earth orbit: Preliminary results. Bull. Amer. Meteor. Soc., 77, 19-40, https://doi.org/10.1175/1520-0477 (1996) $077<0019:$ GSOTAF $>2.0 . C O ; 2$.

Winning, T. E., Y.-L. Chen, and F. Xie, 2017: Estimation of the marine boundary layer height over the central North Pacific using GPS radio occultation. Atmos. Res., 183, 362-370, https://doi.org/10.1016/j.atmosres.2016.08.005.

Wood, R., and C. S. Bretherton, 2004: Boundary layer depth, entrainment, and decoupling in the cloud-capped subtropical and tropical marine boundary layer. J. Climate, 17, 3576-3588,
https://doi.org/10.1175/1520-0442(2004)017<3576:BLDEAD> 2.0.CO;2.

—, and Coauthors, 2011: The VAMOS Ocean-Cloud-Atmosphere-Land Study Regional Experiment (VOCALS-REx): Goals, platforms, and field operations. Atmos. Chem. Phys., 11, 627-654, https://doi.org/10.5194/acp-11-627-2011.

Xie, F., S. Syndergaard, E. R. Kursinski, and B. M. Herman, 2006: An approach for retrieving marine boundary layer refractivity from GPS occultation data in the presence of superrefraction. J. Atmos. Oceanic Technol., 23, 1629-1644, https://doi. org/10.1175/JTECH1996.1.

- , D. L. Wu, C. O. Ao, and A. J. Mannucci, 2010: Atmospheric diurnal variations observed with GPS radio occultation soundings. Atmos. Chem. Phys., 10, 6889-6899, https:// doi.org/10.5194/acp-10-6889-2010.

,,,$----{ }_{-}$, and E. R. Kursinski, 2012: Advances and limitations of atmospheric boundary layer observations with GPS occultation over southeast Pacific Ocean. Atmos. Chem. Phys., 12, 903-918, https://doi.org/10.5194/acp-12-903-2012.

Yu, X., F. Xie, and C. O. Ao, 2018: Evaluating the lower-tropospheric COSMIC GPS radio occultation sounding quality over the Arctic. Atmos. Meas. Tech., 11, 2051-2066, https:// doi.org/10.5194/amt-11-2051-2018.

Zhang, J. A., R. F. Rogers, D. S. Nolan, and F. D. Marks, 2011: On the characteristic height scales of the hurricane boundary layer. Mon. Wea. Rev., 139, 2523-2535, https://doi.org/10. 1175/MWR-D-10-05017.1.

Zhang, Y., L. Wang, J. A. Santanello Jr, Z. Pan, Z. Gao, and D. Li, 2020: Aircraft observed diurnal variations of the planetary boundary layer under heat waves. Atmos. Res., 235, 104801, https://doi.org/10.1016/j.atmosres.2019.104801.

Zhou, C., J. Wang, A. Dai, and P. W. Thorne, 2021: A new approach to homogenize global subdaily radiosonde temperature data from 1958 to 2018. J. Climate, 34, 1163-1183, https://doi.org/10.1175/JCLI-D-20-0352.1.

Zhou, L., 2021: Diurnal asymmetry of desert amplification and its possible connections to planetary boundary layer height: A case study for the Arabian Peninsula. Climate Dyn., 56, 3131-3156, https://doi.org/10.1007/s00382-021-05634-x. 